\title{
Phase II study of pazopanib in combination with paclitaxel in patients with metastatic melanoma
}

\author{
John P. Fruehauf ${ }^{1}$ (I) $\cdot$ Monica El-Masry ${ }^{1} \cdot$ Katherine Osann $^{1} \cdot$ Basmina Parmakhtiar $^{1} \cdot$ Maki Yamamoto $^{2}$. \\ James G. Jakowatz ${ }^{2}$
}

Received: 28 May 2018 / Accepted: 12 June 2018 / Published online: 25 June 2018

(c) The Author(s) 2018

\begin{abstract}
Purpose This phase II study evaluated the safety and clinical activity of pazopanib, a potent and mutlitargeted tyrosine kinase inhibitor (TKI) of vascular endothelial growth factor receptors (VEGFRs)-1, -2 and -3, platelet-derived growth factor receptor (PDGFR)- $\alpha$ and $\beta$, and cKit, in combination with metronomic paclitaxel in patients with metastatic melanoma. Experimental design Sixty chemotherapy-naive patients received pazopanib at a starting dose of $800 \mathrm{mg}$ daily in combination with metronomic dosing of paclitaxel $80 \mathrm{mg} / \mathrm{m}^{2}$ weekly thrice every 4 weeks. The primary endpoint was 6-month progression-free survival (PFS) rate, while secondary endpoints included 1-year overall survival rate, RECIST response rates, progression-free survival rates and median overall survival. Prior BRAF-targeted therapy or checkpoint inhibitors were permitted.

Results The 6-month PFS rate was $68 \%$, with a 1 -year OS rate of $48 \%$. Objective response rate was $37 \%$ comprising one complete and 20 partial responses. Stable disease at 8 weeks was noted in 32 patients (55\%) with an overall clinical benefit rate of 93\%. Six-month median progression-free survival was 8 months and median OS was 12.7 months. The most frequently $(>15 \%)$ reported non-hematologic, treatment-related adverse events were fatigue, diarrhea, hypertension, transaminitis and peripheral neuropathy. Treatment-related non-fatal bowel perforation, a known class effect, occurred in one patient. No significant association was noted between plasma levels of pazopanib and response.

Conclusions The combination of pazopanib and metronomic paclitaxel was well-tolerated, demonstrating significant activity in metastatic melanoma. Further evaluation of this combination is warranted.
\end{abstract}

Keywords Pazopanib $\cdot$ Metronomic paclitaxel $\cdot$ Metastatic melanoma $\cdot$ Antiangiogenic agent $\cdot$ BRAF

\section{Introduction}

The National Cancer Institute estimates that 87,110 new cases of melanoma will be diagnosed and 9730 deaths from the disease will occur in the United States in 2017 [1]. While surgery is often curative with early-stage melanoma, metastatic melanoma has had a median survival time

John P. Fruehauf

jfruehau@uci.edu

1 Division of Hematology/Oncology, Chao Family Comprehensive Cancer Center, University of California, 101 The City Drive South, Bldg 56, Orange, Irvine, CA 92868 , USA

2 Division of Surgical Oncology, Chao Family Comprehensive Cancer Center, University of California, 101 The City Drive South, Bldg 56, Orange, Irvine, CA 92868, USA of only 6-9 months [2]. However, the treatment paradigm for patients with stage IV melanoma has changed in recent years leading to improved survival.

Current therapies for advanced melanoma typically include BRAF (BRaf proto-oncogene, serine/threonine kinase) and MEK (mitogen-activated protein kinase)targeted agents and/or checkpoint-targeted immunotherapy (ipilimumab, pembrolizumab, and nivolumab). Both approaches have been shown to extend survival [3]. Combinations of BRAF and MEK inhibitors, dabrafenib plus tramietinib and vemurafeniib plus cobimetinib, have demonstrated 60-70\% response rates, median PFS of 11 months and median OS of 25.1 months [4-6].

With respect to the checkpoint inhibitors, ipilimumab which targets T cell CTLA-4, was the first agent in this class to show an improved OS in melanoma, and meta-analysis of pooled data from ipilimumab trials which included 1861 
melanoma patients revealed a 3-year OS rate of $22 \%[7,8]$. The PD- 1 targeted agents pembrolizumab and nivolumab were subsequently shown to be effective in melanoma [3].

In spite of the great advance offered by BRAF/MEK and checkpoint inhibitors, PFS remains below 1 year for both classes of therapy and median OS is approximately 24 months. There is an unmet need for a second- or thirdline therapy that could offer further incremental improvements in PFS and OS.

Tumor angiogenesis, mediated by the vascular endothelial growth factor (VEGF) signaling network, is strongly implicated in melanoma progression [9-11]. In patients with melanoma, elevated levels of VEGF are associated with poor outcome [12]. Additionally, preclinical studies have shown that simultaneous inhibition of VEGF receptors (VEGFRs)-1 and -2, but not the sole inhibition of either receptor, blocked melanoma growth and metastasis [13]. Taken together, these findings strongly suggest the involvement of VEGFR signaling pathways in melanoma and support the hypothesis that VEGF-targeted antiangiogenesis therapy (AAT) may prove to be effective either alone or in combination with other therapies.

The benefits of AAT have been shown in two phase II trials for advanced-stage melanoma [14, 15]. The first trial evaluated the activity of axitinib, a selective inhibitor of VEGR-1, -2 and -3 [14]. For the 32 patients enrolled, the objective response rate (RR) was $18.8 \%$, comprising one complete response and five partial responses with a median response duration of 5.9 months $(95 \% \mathrm{CI}$, 5.0-17.0). Stable disease at 16 weeks was noted in six patients (18.8\%), with an overall clinical benefit rate of $37.5 \%$. Six-month progression-free survival (PFS) was $33.9 \%$, 1-year overall survival was $28.1 \%$, and median overall survival was 6.6 months. While these data suggested moderate single-agent activity, combining antiangiogenesis agents with chemotherapy was hypothesized to be more effective. The combination of carboplatin and paclitaxel (CP) alone was subsequently evaluated versus CP plus bevacizumab (CPB) [15]. Two hundred fourteen patients (73\% with M1c disease) were randomly assigned. With a median follow-up of 13 months, median PFS was 4.2 months for the CP arm and 5.6 months for the CPB arm (HR, $0.78 ; P=.1414)$. Overall RRs were 16.4 and $25.5 \%$, respectively $(P=.1577)$. With 17 -month follow-up, median OS was 9.2 versus 12.3 months, respectively (HR, $0.79 ; P=.1916)$. No new safety signals were observed.

These results indicated that AAT was active in advanced melanoma and that it could be combined with chemotherapy. We, therefore, designed and initiated a phase II trial evaluating daily oral pazopanib, a potent and mutlitargeted TKI of vascular endothelial growth factor receptors (VEGFRs)-1, -2, and -3, in combination with paclitaxel in patients with metastatic melanoma [16]. This combination was selected based in part on favorable phase I safety and pharmacokinetic data [17]. We employed a metronomic schedule for paclitaxel based on preclinical and clinical data indicating that metronomic administration enhances paclitaxel's antiangiogenesis effects [18-20].

\section{Materials and methods}

\section{Patients}

Patients $\geq 18$ years of age with histologically confirmed advanced melanoma who were chemotherapy-naive were eligible for enrollment. Previous cytokine, immunotherapy or BRAF-targeted therapy was permitted, but had to be completed 28 days prior to first dose of study medication. Other eligibility criteria included measurable disease based on Response Evaluation Criteria in Solid Tumors (RECIST), adequate major organ function, Eastern Cooperative Oncology Group performance status of 0 or 1 , and informed consent. Patients were excluded if they met any of the following criteria: previous treatment with antiangiogenic agents, preexisting uncontrolled hypertension, i.e., systolic blood pressure (BP) $>150 \mathrm{~mm} \mathrm{Hg}$ and diastolic $\mathrm{BP}(\mathrm{dBP})>90 \mathrm{~mm} \mathrm{Hg}$, active seizure disorder, and major surgical procedure or radiation therapy within 4 weeks of treatment. Prior central nervous system (CNS) metastases was allowed for subjects who had previously treated CNS metastases (surgery \pm radiotherapy, radiosurgery, or gamma knife) and were asymptomatic, had no clinical evidence of active CNS metastases for $\geq 28$ days prior to enrollment and had no requirement for steroids or enzyme-inducing anticonvulsants (EIAC). Patients with a recent history (6 months) of myocardial infarction or myocardial disease requiring stenting or angioplasty, or heart failure associated with ejection fractions below $50 \%$ were excluded.

\section{Study design}

This was an open-label, phase II trial of the clinical activity, safety, and tolerability of pazopanib plus metronomic paclitaxel in patients with unresectable stage III or metastatic melanoma. The primary objective was to determine the 6-month progression-free survival (PFS) rate. Secondary endpoints included the 1- and 2-year survival rates, median progression-free survival, median overall survival, objective response rate, and clinical benefit rate $(\mathrm{SD}+\mathrm{PR}+\mathrm{CR})$. This study was approved by the institutional review board and was carried out in accordance with the International Conference on Harmonization Good Clinical Practice guidelines 
protocol. Written informed consent was obtained prior to patients entering the study. The study was registered at ClinicalTrials.gov (NCT01107665).

\section{Study treatment}

All subjects enrolled received pazopanib $800 \mathrm{mg}$ daily dosing in combination with a metronomic dose of paclitaxel $80 \mathrm{mg} / \mathrm{m}^{2}$ weekly for 3 weeks every 4 weeks. Subjects were permitted to receive full supportive care during the study, including transfusion of blood and blood products, treatment with antibiotics, anti-emetics, anti-diarrheal agents, analgesics, erythropoietin, colony stimulating factors or bisphosphonates when appropriate.

Study treatment continued until subjects experienced disease progression, death, unacceptable toxicity, or withdrawal of consent for any other reasons. Treatment was interrupted in patients with $\mathrm{AE}$ grade $\geq 2$ that was not controlled by supportive medication and was resumed at the same dose after resolution to grade 1 or baseline levels. Treatment was resumed at a $20 \%$ lower dose after resolution to grade 1 or baseline levels for non-hematologic AEs grade $\geq 3$, grade 4 hematologic AEs, or recurrent subjectively intolerable toxicity. At each visit during the treatment period, subjects were evaluated for the occurrence of AEs and laboratory abnormalities. Dose adjustments were implemented whenever clinically indicated. If dose reduction was necessary, two dose reductions for pazopanib were permitted in a stepwise fashion (initially to $600 \mathrm{mg}$ and subsequently to $400 \mathrm{mg}$ if necessary) to achieve resolution of toxicity to grade 1 or baseline. If the toxicity did not recur or worsen, the dose was then increased stepwise back to 600 and $800 \mathrm{mg}$ after monitoring for 10-14 days at each step if toxicity did not recur or worsen. Dose interruptions also occurred for uncontrolled elevated BP, hemoptysis, or proteinuria.

For patients experiencing $\geq$ grade 3 toxicity attributed to paclitaxel, an initial dose reduction to $65 \mathrm{mg} / \mathrm{m}^{2}$ was implemented. Upon recovery, the paclitaxel dose could be re-escalated to $80 \mathrm{mg} / \mathrm{m}^{2}$ weekly at the discretion of the investigator. Subjects requiring frequent omissions or dose reductions for individual infusions (eg, one-half of administered doses during a two-cycle period), were allowed to discontinue treatment with paclitaxel and resume therapy with pazopanib monotherapy per investigator discretion. Patients deriving clinical benefit could continue to receive treatment after meeting criteria for study completion.

\section{Study assessments}

Baseline screening with CT CAP and MRI of the brain, ECHO cardiograms and ECG was required. WB PET/ CT could be carried out in lieu of CT CAP. Tumors were measured using computed tomography $(\mathrm{CT})$ or magnetic resonance imaging at least every 8 weeks. RECIST 1.1 was utilized for response determinations. Blood samples were collected on day 1 (pre-dose) and every 4 weeks thereafter for analysis of blood counts, complete metabolic profile including LDH. A thyroid panel, urine spot creatinine and protein as well as amylase and lipase were monitored every 8 weeks.

\section{Analysis of blood-based pazopanib}

Plasma samples for assay of serum pazopanib levels were collected C1D1 (pre-dose), C2D1 and C2D28.

\section{Statistical methods}

The study was conducted using a two-stage Simon Minimax design [21]. Due to lower response rates to conventional chemotherapy for this indication, the $\mathrm{p} 0$ and $\mathrm{p} 1$ were set at 5 and 20\%, respectively. The $\alpha$ and $\beta$ error rates were set at 0.10 and 0.10 , respectively. These criteria resulted in a sample size of 18 patients in stage 1 and a minimum of 14 patients in stage 2 (based on Power Analysis and Sample Size 2002 software, Kaysville, UT). At least one confirmed response (i.e., PR or $\mathrm{CR}$ ) was needed in stage 1 to allow expansion of the trial to stage 2 . Safety and efficacy analyses included all patients who received at least one dose of pazopanib and paclitaxel and had a baseline assessment of disease. Patients who died, progressed, or discontinued treatment prior to experiencing a CR or PR were classified as non-responders. An analysis for constructing the historical control 6-month PFS rate (performance status, presence of visceral disease, brain metastases and gender) was performed based on the calculation method described by Korn et al. [22]. Statistical analysis was performed using GraphPad Prism, GraphPad Software, Inc.

\section{Results}

\section{Patient characteristics}

60 patients were enrolled in the study and received at least one dose of pazopanib and paclitaxel. Patient baseline characteristics are summarized in Table 1. Two patients had unresectable stage III disease, while 58 were stage IV. Twenty-three patients (38\%) had received prior systemic treatment for any disease stage. Eleven patients $(18 \%)$ had prior checkpoint inhibitor treatment, while only four patients (7\%) had prior BRAF-targeted therapy. One patient had received adjuvant temozolomide. Lung and liver were the most common metastatic disease sites. CNS involvement was present at baseline in eight patients (13\%). Thirty-five patients (58\%) were classified as M1C at baseline. 
Table 1 (a) Patient characteristics at baseline and (b) prior therapy

\begin{tabular}{ll}
\hline & Pazopanib $(N=60)$ \\
\hline (a) & \\
Median age, years & 64 \\
Range & $36-90$ \\
Sex, $n(\%)$ & \\
Male & $45(75)$ \\
Female & $15(25)$ \\
ECOG performance status, $n(\%)$ & \\
0 & $49(82)$ \\
1 & $11(18)$ \\
Metastatic stage, $n(\%)$ & \\
III & $2(3)$ \\
M1A & $6(10)$ \\
M1B & $17(28)$ \\
M1C & $35(58)$ \\
Common metastatic sites, $n(\%)$ & \\
Lung & $29(48)$ \\
Liver & $12(20)$ \\
CNS & $8(13)$ \\
BRAF mutant positive & $14(23)$ \\
(b) & \\
Prior therapy, $n(\%)$ & \\
Any & \\
HD IFN & $23(38)$ \\
Pembrolizumab & $11(18)$ \\
BRAF inhibitor & $9(15)$ \\
Vaccine & $2(3)$ \\
\hline & \\
&
\end{tabular}

The median duration of treatment was 10.6 months (range, 0.4-42.3) with 20 patients (33\%) receiving therapy for $\geq 12$ months. Treatment discontinuation was related to adverse events/complications in $6(10 \%)$ patients, death on study in 1 patient (1.7\%), disease progression during active treatment in 43 (72\%), discontinuation of treatment for other complicating disease state $3(5 \%)$, patient withdrawal or refusal after beginning protocol therapy $5(8.3 \%)$ and treatment completed per protocol criteria $1(1.7 \%)$. One patient remains on active treatment with pazopanib and paclitaxel $(1.7 \%)$.

\section{Clinical activity}

The ORR was 37\% comprising one CR (2\%) and twenty PRs (34\%) (Table 2). The maximum percentage change in target lesion size is shown in Fig. 1. Thirty-two patients (55\%) had a best response of stable disease at least 8 weeks in duration, yielding an overall clinical benefit rate (percentage of patients with a best response $\geq$ stable disease) of $91 \%$. An additional four patients (7\%) had progressive disease.
Median PFS was 8 months (95\% CI shown on Kaplan Myer Plot) (Fig. 2a), and median OS was 12.7 months (95\% CI shown on Kaplan Myer Plot) (Fig. 2b). Six-month PFS was $68 \%$ and 1-year OS was $48.1 \%$ and 2-year OS was $27 \%$. The response profile for the BRAF WT subset $(n=44)$ was 1 (2\%) CR, 12 (27\%) PR, 28 (64\%) SD and 3 (7\%) PD. For the BRAF V600E-positive cases $(n=14)$ there were $8(57 \%)$ PR, $5(36 \%)$ SD and 1 (7\%) PD. While there was a trend towards improved OS in the BRAF mutant subset (18 months) versus the BRAF WT/unknown group (11.3 months), this was not statistically significant (HR $0.78495 \%$ CI $0.41-1.54$, Log Rank $P=.49$ ).

\section{Safety}

The most frequently ( $>15 \%)$ reported non-hematologic, treatment-related AEs included fatigue, hypertension, hoarseness, and diarrhea, (Table 3). The majority of these events were grade $1 / 2$. The most common grade $\geq 3 \mathrm{AE}$ was transaminitis $(n=20 ; 33 \%)$ followed by fatigue $(n=8 ; 13 \%)$ and hypertension $(n=8 ; 13 \%)$. There was one case of bowel perforation requiring surgery $(n=1 ; 1.7 \%)$. There were 9 patients who had dose reductions in pazopanib $9(15 \%), 42$ (70\%) who had treatment interrupted, and therapy discontinued in $10(16.7 \%)$ cases.

\section{PK analysis}

Pharmacokinetic analysis of pazopanib blood levels failed to yield a significant relationship with response (Fig. 3). This finding parallels with that seen for axitinib in melanoma [14].

\section{Discussion}

These results demonstrate that pazopanib plus metronomic paclitaxel exhibited significant activity in this group of patients with advanced stage melanoma, of which 58\% fell into the poor prognosis stage M1C (Table 1). Antitumor activity was observed with an ORR of $37 \%$, including one CR and twenty PRs, with a median PFS duration of 8 months (Table 3 ). An additional 32 patients (55\%) experienced stable disease lasting at least 8 weeks. Although cross-study comparisons are complicated by methodological differences, this response rate is superior to the $8-13 \%$ ORR provided by standard DTIC or temozolomide therapy for advanced disease and exceeds the ORR range of 10-20\% associated with interferon- $\alpha$ and IL- 2 or carboplatin and paclitaxel combination therapy [23-26]. Additionally, the 6-month PFS rate was 68\%, with a 1-year OS rate was $48.1 \%$ (Fig. 2). Median OS was 12.7 months (95\% CI shown on 


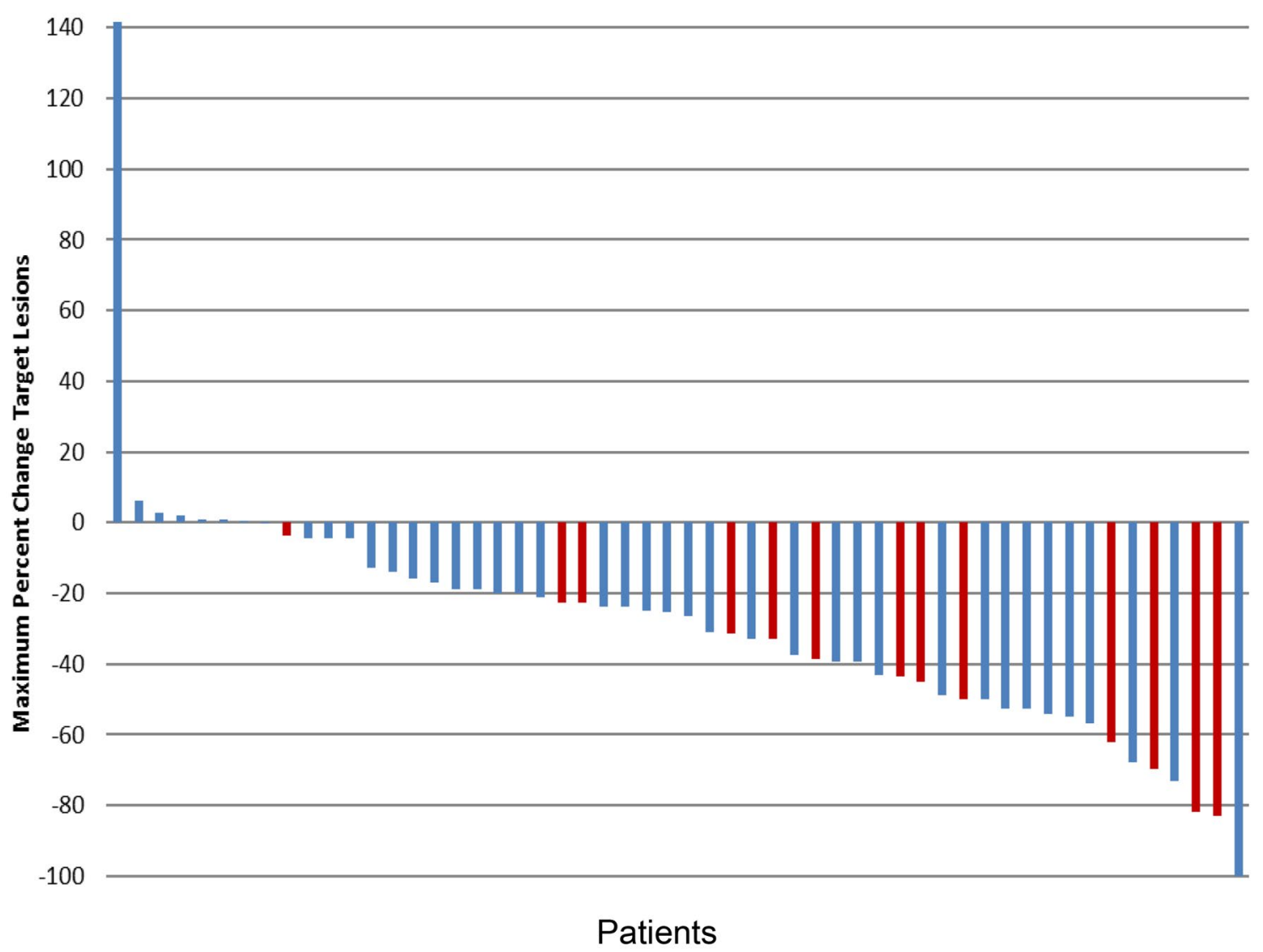

Fig. 1 Maximum percentage change in target lesion size, based on response evaluation criteria 1.1 in solid tumors $(n=54$; six were invaluable for response). BRAF mutation-positive cases shown in red bars

Kaplan-Meier Plot). No clear association was noted between pazopanib serum levels and response (Fig. 3).

Current first-line therapy for stage IV melanoma generally includes either a checkpoint inhibitor or a BRAF/MEK tyrosine kinase inhibitor combination for patient's bearing tumors that carry a V600E or V600K BRAF mutation. In the clinical trial that compared the checkpoint inhibitor pembrolizumab to investigator choice chemotherapy (ICC) in patient's refractory to ipilimumab or to prior BRAF-targeted therapy, pembrolizumab was superior to ICC with respect to PFS and tolerability, providing the basis for accelerated approval in advanced melanoma [27]. In a subsequent head to head comparison, pembrolizumab given every 3 weeks demonstrated greater efficacy than ipilimiumab, with a 6-month PFS rate of $46.4 \%$, and a 12 -month survival rate of $68.4 \%$ [28]. In updated analysis, pembrolizumab-treated patients had a RR of $36.1 \%$, with a $55.3 \%$ 24-month survival rate [29]. These response rate results are similar to those we observed with pazopanib plus paclitaxel, although the 24-month survival rate was lower for pazopanib and paclitaxel at $27 \%$.

Nivolumab, the second PD-1-targeting checkpoint inhibitor approved for melanoma, was compared to investigator choice chemotherapy (ICC) as second- or later-line treatment in patients who were refractory to ipilimumab or BRAF agents. Confirmed objective responses were seen in 31.7 versus $10.6 \%$, respectively [30]. These results were further corroborated in previously untreated patients without a BRAF mutation, where nivolumab was compared to dacarbazine [31]. Patients in the nivolumab arm showed a response rate of 40.0 versus $13.9 \%$ in the dacarbazine arm. Nivolumab-treated patients had a median PFS of 5.1 months and a 1-year survival rate of $72.9 \%$. This finding is also similar to the results reported here for pazopanib plus paclitaxel which demonstrated a PFS of 8 months.

The combination of nivolumab with ipilimumab has also been found to be active. The 3-armed phase III study randomized 945 treatment-naive patients with unresectable or metastatic 1:1:1 to nivolumab plus ipilimumab, nivolumab alone, or ipilimumab alone [32]. The combination showed a response rate of 58 versus $44 \%$ for nivolumab alone, and $19 \%$ for ipilimumab alone. The combination group had a median PFS of 11.5 months. The clinical benefit provided by the combination was most evident in patients with PD-L1-negative tumors. However, the combination arm 
A
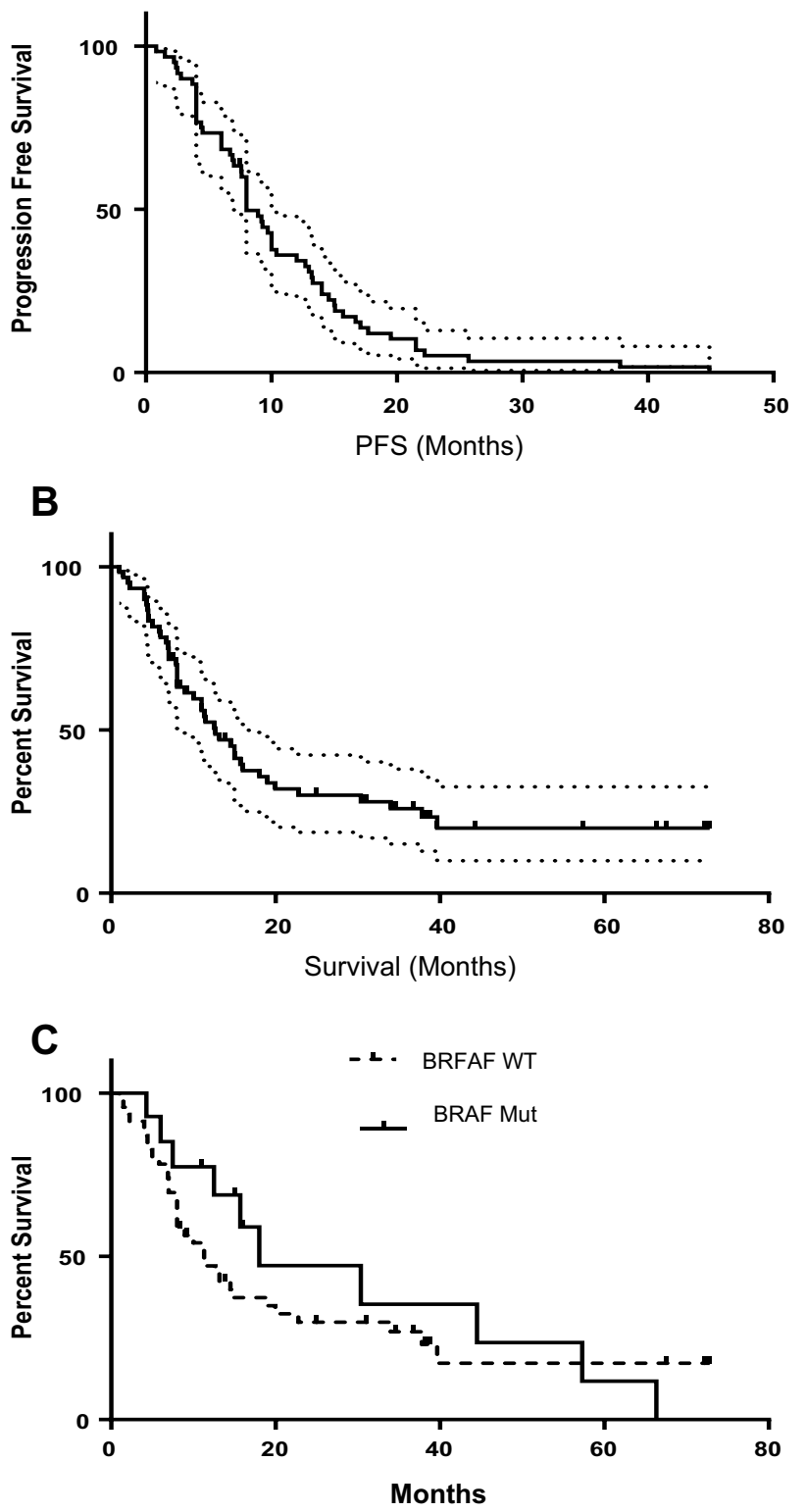

Fig. 2 Kaplan-Meier estimates $( \pm 95 \% \mathrm{CI})$ of a progression-free survival in all patients $(n=60)$; and $\mathbf{b}$ overall survival $(n=60)$; and $\mathbf{c}$ comparison of OS between BRAF WT/unknown $(n=46)$ and BRAF mutation-positive patients $(n=14)$

experienced a high frequency of severe immune-related adverse events with almost all patients reporting at least one side effect, while $57 \%$ showed an event of grade 3 or 4 . The combination therapy had to be stopped in $39 \%$ of all patients due to side effects such as diarrhea with colitis or hepatitis with elevated liver enzymes.

BRAF inhibition can also be considered as first-line therapy for patients with V600E of V600K mutations. Combinations of BRAF and MEK inhibitors, dabrafenib plus trametinib or vemurafeniib plus cobimetinib, have
Table 2 Response rates $N=58$ evaluable

\begin{tabular}{ll}
\hline ORR & $21(36 \%)$ \\
DCR $(\mathrm{CR}+\mathrm{PR}+\mathrm{SD})$ & $53(91 \%)$ \\
$\mathrm{CR}$ & $1(2 \%)$ \\
$\mathrm{PR}$ & $20(34 \%)$ \\
$\mathrm{SD}$ & $32(55 \%)$ \\
$\mathrm{PD}$ & $4(7 \%)$ \\
\hline
\end{tabular}

Table 3 Safety findings: non-hematologic, treatment-related adverse events (AEs) reported by at least $15 \%$ of patients or of clinical interest, and hematologic abnormalities

\begin{tabular}{lll}
\hline & \multicolumn{2}{l}{ Number of patients, $N=32 n(\%)$} \\
\cline { 2 - 3 } & Total $^{\mathrm{a}}$ & Grade $3 / 4^{\mathrm{a}}$ \\
\hline Non-hematologic AEs & $44(73)$ & $8(13)$ \\
Fatigue & $37(62)$ & $3(5)$ \\
Diarrhea $^{\mathrm{b}}$ & $35(48)$ & $1(2)$ \\
Hypertension $^{\mathrm{b}}$ & $29(48)$ & $1(2)$ \\
Nausea & $21(35)$ & $10(17)$ \\
Transaminitis $_{\text {Hematologic abnormalities }}{ }^{\mathrm{c}}$ & & 0 \\
Anemia & $13(22)$ & 0 \\
Thrombocytopenia & $4(7)$ & $9(15)$ \\
Neutropenia & $18(30)$ & \\
\hline a National Cancer Institute & Common Terminology & Criteria for \\
Adverse Events, version 3.0 & & \\
${ }^{b}$ Not otherwise specified & & \\
${ }^{c}$ Based on laboratory data & &
\end{tabular}

demonstrated $60-70 \%$ response rates, median PFS of 11 months and median OS of 25.1 months [4-6]. While the BRAF mutant subset in this trial was limited in size $(n=14)$, the response rate to pazopanib plus paclitaxel was $57 \%$ with a median OS of 18 months. Pazopanib does exhibit activity against both WT and V600E mutant BRAF, with an apparent inhibitory concentration of 68 and $160 \mathrm{nM}$, respectively (Investigator Brochure). Cmax levels of pazopanib are in the order of $130 \mu \mathrm{M}$, suggesting that the levels of pazopanib achieved at standard doses can exhibit tyrosine kinase inhibition (TKI) activity against both WT and mutant BRAF. This pharmacodynamic effect of pazopanib may explain the higher RR of $57 \%$ we observed for the BRAF subset.

The results of our trial indicate that the pazopanib plus paclitaxel combination can be given safely to patients with advanced melanoma and that its clinical activity is similar to that observed for current first-line treatment. This antiangiogenesis regimen may be of value for patients who fail standard first- and or second-line therapy, offering an additional incremental benefit to patients with advanced melanoma. Further exploration of this regimen is warranted. 


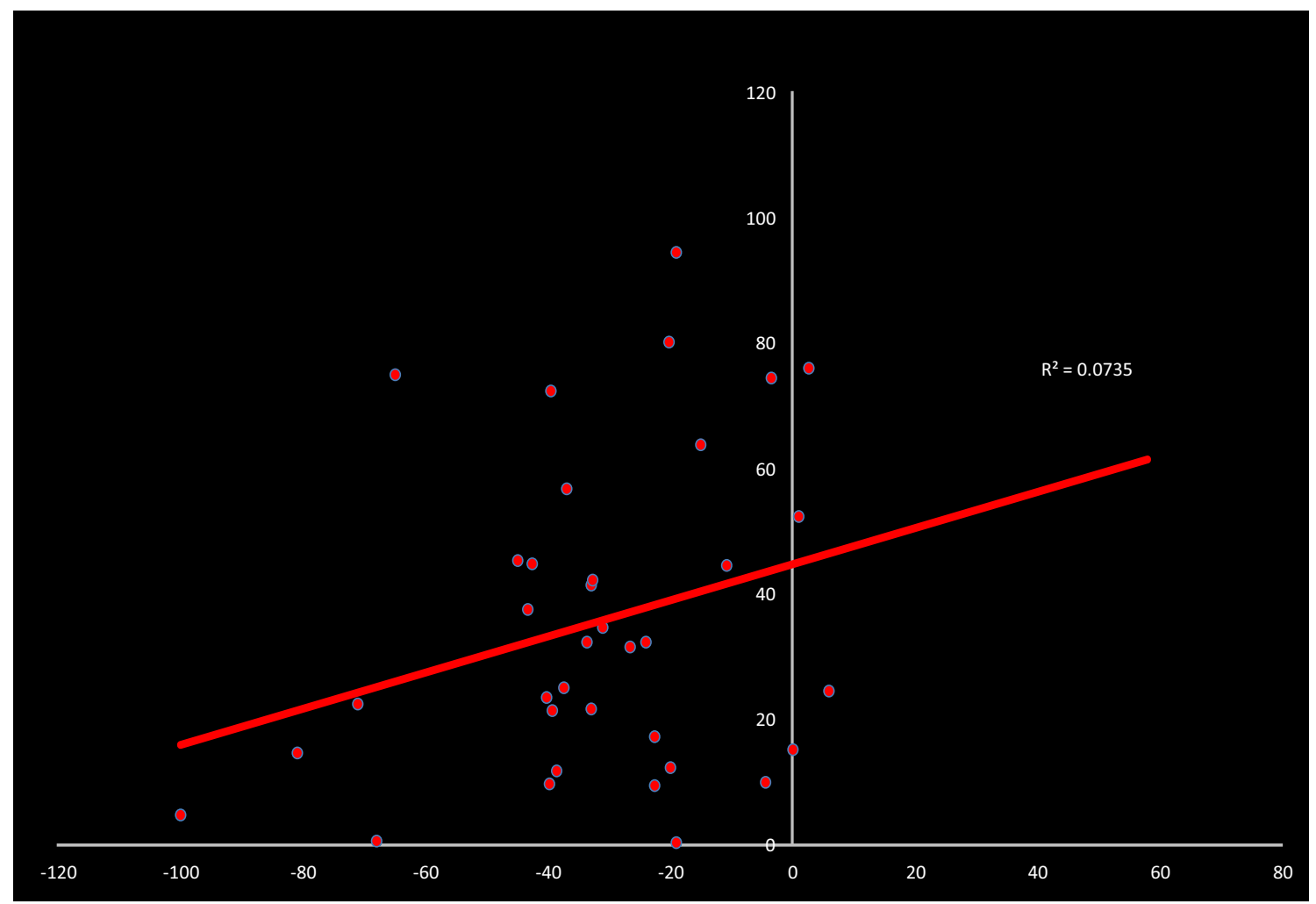

Fig. 3 Correlation coefficient between pazopanib pre-dose blood levels on C2D1 and maximum percent change in target lesions

Acknowledgements We would like to thank all of the participating patients and their families, as well as the investigators, research nurses, study coordinators, and operations staff. We gratefully acknowledge pharmacokinetic analyses by Glaxo Smith Kline.

Author contributions The authors thank Beverly Alger, David Hsiang, Leonard Sender, Kevin Kong, Claudette Bettis, Slomit Ein-Gal, Cameron Casey, Cynthia Mann, Jinah Chung, Tu Kersavage, Natalie Ayala and Marlene S. Alvarez for their contributions.

\section{Compliance with ethical standards}

Conflict of interest Research support: Glaxo Smith Kline, Inc. and Novartis, Inc. JP Fruehauf has received financial support from Novartis for speaking at educational symposia and for developing slide decks for educational symposia. No other authors have conflicts of interest.

Ethical standards This study was performed in accordance with the ethical standards as laid down in the 1964 Declaration of Helsinki and its later amendments or comparable ethical standards. The IND for this FDA approved trial is held by JP Fruehauf and UC Irvine.

Informed consent This trial was carried out with the approval of the UC Irvine IRB and all patients were required to provided informed consent prior to their participation.

Open Access This article is distributed under the terms of the Creative Commons Attribution 4.0 International License (http://creat ivecommons.org/licenses/by/4.0/), which permits unrestricted use, distribution, and reproduction in any medium, provided you give appropriate credit to the original author(s) and the source, provide a link to the Creative Commons license, and indicate if changes were made.

\section{References}

1. Siegel RL, Miller KD, Jemal A (2016) Cancer statistics, 2017. CA Cancer J Clin 67:7-30

2. Balch CM, Soong SJ, Gershenwald JE, Thompson JF, Reintgen DS, Cascinelli N et al (2001) Prognostic factors analysis of 17,600 melanoma patients: validation of the American Joint Committee on Cancer melanoma staging system. J Clin Oncol 19:3622-3634

3. Heppt MV, Dietrich C, Graf SA, Ruzicka T, Tietze JK, Berking $\mathrm{C}$ (2016) The systemic management of advanced melanoma in 2016. Oncol Res Treat 39:635-642

4. Long GV, Weber JS, Infante JR, Kim KB, Daud A, Gonzalez R, Sosman JA, Hamid O, Schuchter L, Cebon J, Kefford RF, Lawrence D, Kudchadkar R, Burris HA 3rd, Falchook GS, Algazi A, Lewis K, Puzanov I, Ibrahim N, Sun P, Cunningham E, Kline AS, Del Buono H, McDowell DO, Patel K, Flaherty KT (2016) Overall survival and durable responses in patients with BRAF V600-mutant metastatic melanoma receiving dabrafenib combined with trametinib. J Clin Oncol 34(8):871-878

5. Flaherty K, Davies MA, Grob JJet al (2016) Genomic analysis and 3-y efficacy and safety update of COMBI-d: a phase 3 study of dabrafenib (D) + trametinib (T) vs D monotherapy in patients (pts) with unresectable or metastatic BRAF V600E/K-mutant 
cutaneous melanoma. J Clin Oncol 34:9502-9502 (suppl; abstr 9502)

6. Larkin J, Ascierto PA, Dréno B, Atkinson V, Liszkay G, Maio M, Mandalà M, Demidov L, Stroyakovskiy D, Thomas L, de la CruzMerino L, Dutriaux C, Garbe C, Sovak MA, Chang I, Choong N, Hack SP, McArthur GA, Ribas A (2014) Combined vemurafenib and cobimetinib in BRAF-mutated melanoma. N Engl J Med 371:1867-1876

7. Hodi FS, O'Day SJ, McDermott DF, Weber RW, Sosman JA, Haanen JB et al (2010) Improved survival with ipilimumab in patients with metastatic melanoma. N Engl J Med 363:711-723

8. Schadendorf D, Hodi FS, Robert C, Weber JS, Margolin K, Hamid O, Patt D, Chen TT, Berman DM, Wolchok JD (2015) Pooled analysis of long-term survival data from phase II and phase III trials of ipilimumab in unresectable or metastatic melanoma. Journal Clin Oncol 9:JCO-2014

9. Jour G, Ivan D, Aung PP (2016) Angiogenesis in melanoma: an update with a focus on current targeted therapies. J Clin Pathol 69:472-483

10. Grant SW, Kyshtoobayeva AS, Kurosaki T, Jakowatz J, Fruehauf JP (1998) Mutant p53 correlates with reduced expression of thrombospondin-1, increased angiogenesis, and metastatic progression in melanoma. Cancer Detect Prev 22:185-94

11. Einspahr JG, Thomas TL, Saboda K, Nickolof BJ, Warneke J, Curiel-Lewandrowski C, Ranger-Moore J, Duckett L, Bangert J, Fruehauf JP, Alberts DS (2007) Expression of vascular endothelial growth factor in early cutaneous melanocytic lesion progression. Cancer 110:2519-2527

12. Ugurel S, Rappl G, Tilgen W, Reinhold U (2001) Increased serum concentration of angiogenic factors in malignant melanoma patients correlates with tumor progression and survival. J Clin Oncol 19:577-583

13. Gille J, Heidenreich R, Pinter A, Schmitz J, Boehme B, Hicklin DJ et al (2007) Simultaneous blockade of VEGFR-1 and VEGFR-2 activation is necessary to efficiently inhibit experimental melanoma growth and metastasis formation. Int J Cancer 120:1899-1908

14. Fruehauf JP, Lutzky J, McDermott DF, Brown CK, Meric J-B, Rosbrook B, Shalinsky DR, Liau KF, Niethammer AG, Kim S, Rixe O (2011) Multicenter, phase II study of axitinib, a selective second-generation inhibitor of vascular endothelial growth factor receptors 1, 2, 3, in patients with metastatic melanoma. Clin Cancer Res 17:7462-7469

15. Kim KB, Sosman JA, Fruehauf JP, Linette GP, Markovic SN, McDermott DF, Weber JS, Nguyen H, Cheverton P, Chen D, Peterson AC, Carson WE 3rd, O'Day SJ (2012) BEAM: a randomized phase II study evaluating the activity of bevacizumab in combination with carboplatin plus paclitaxel in patients with previously untreated advanced melanoma. J Clin Oncol 30:34-41

16. Harris PA, Boloor A, Cheung M et al (2008) Discovery of 5-[[4-[(2,3-Dimethyl-2H-indazol-6-yl)methylamino]-2-pyrimidinyl]amino]-2-methyl-benzenesulfonamide (Pazopanib), a novel and potent vascular endothelial growth factor receptor inhibitor. J Med Chem 51(15):4632-4640

17. Hurwitz HI, Dowlati A, Saini S, Savage S, Suttle AB, Gibson DM, Hodge JP, Merkle EM, Pandite L (2009) Phase I trial of pazopanib in patients with advanced cancer. Clin Cancer Res 15:4220-4227

18. Bocci G, Di Paolo A, Danesi R (2013) The pharmacological bases of the antiangiogenic activity of paclitaxel. Angiogenesis $16: 481-492$
19. Kerbel RS (2007) Improving conventional or low dose metronomic chemotherapy with targeted antiangiogenic drugs. Cancer Res Treat 39:150-159

20. Pasquier E, Kavallaris M (2010) André N. Metronomic chemotherapy: new rationale for new directions. Nat Rev Clin Oncol $7: 455-465$

21. Simon R (1989) Optimal two-stage designs for phase II clinical trials. Control Clin Trials 10:1-10

22. Korn EL, Liu PY, Lee SJ, Chapman JA, Niedzwiecki D, Suman VJ et al (2008) Meta-analysis of phase II cooperative group trials in metastatic stage IV melanoma to determine progression-free and overall survival benchmarks for future phase II trials. J Clin Oncol 26:527-3427

23. Bedikian AY, Millward M, Pehamberger H, Conry R, Gore M, Trefzer U et al (2006) Bcl-2 antisense (oblimersen sodium) plus dacarbazine in patients with advanced melanoma: the Oblimersen Melanoma Study Group. J Clin Oncol 24:4738-4745

24. Kaufmann R, Spieth K, Leiter U, Mauch C, von den Driesch P, Vogt $\mathrm{T}$ et al (2005) Temozolomide in combination with interferon-alfa versus temozolomide alone in patients with advanced metastatic melanoma: a randomized, phase III, multicenter study from the Dermatologic Cooperative Oncology Group. J Clin Oncol 23:9001-9007

25. Atkins MB, Lotze MT, Dutcher JP, Fisher RI, Weiss G, Margolin K et al (1999) High-dose recombinant interleukin 2 therapy for patients with metastatic melanoma: analysis of 270 patients treated between 1985 and 1993. J Clin Oncol 17:2105-2116

26. Hauschild A, Agarwala SS, Trefzer U, Hogg D, Robert C, Hersey P et al (2009) Results of a phase III, randomized, placebocontrolled study of sorafenib in combination with carboplatin and paclitaxel as second-line treatment in patients with unresectable stage III or stage IV melanoma. J Clin Oncol 27:2823-2830

27. Ribas A, Puzanov I, Dummer R, Schadendorf D, Hamid O, Robert C, Hodi FS, Schachter J, Pavlick AC, Lewis KD, Cranmer LD (2015) Pembrolizumab versus investigator-choice chemotherapy for ipilimumab-refractory melanoma (KEYNOTE-002): a randomised, controlled, phase 2 trial. Lancet Oncol 16:908-918

28. Robert C, Schachter J, Long GV, Arance A, Grob JJ, Mortier L, Daud A, Carlino MS, McNeil C, Lotem M, Larkin J (2015) Pembrolizumab versus ipilimumab in advanced melanoma. N Engl J Med 372:2521-2532

29. Schachter J, Ribas A, Long GV, Arance A, Grob JJ, Mortier L (2016) Pembrolizumab versus ipilimumab for advanced melanoma: final overall survival analysis of KEYNOTE-006. J Clin Oncol 34(suppl):9504

30. Weber JS, D'Angelo SP, Minor D, Hodi FS, Gutzmer R, Neyns B Hoeller C, Khushalani NI, Miller WH, Lao CD, Linette GP (2015) Nivolumab versus chemotherapy in patients with advanced melanoma who progressed after anti-CTLA-4 treatment (CheckMate 037): a randomised, controlled, open-label, phase 3 trial. Lancet Oncol 16:375-384

31. Robert C, Long GV, Brady B, Dutriaux C, Maio M, Mortier L, Hassel JC, Rutkowski P, McNeil C, Kalinka-Warzocha E, Savage KJ (2015) Nivolumab in previously untreated melanoma without BRAF mutation. N Engl J Med 372(4):320-330

32. Larkin J, Chiarion-Sileni V, Gonzalez R, Grob JJ, Cowey CL, Lao CD, Schadendorf D, Dummer R, Smylie M, Rutkowski P, Ferrucci PF (2015) Combined nivolumab and ipilimumab or monotherapy in untreated melanoma. N Engl J Med 373:23-34 